\title{
Uma arquitetura para detecção online de transientes em sinais de eletrocardiograma sobre o protocolo PM-AH
}

Diego Rodrigues de Carvalho*, Bruno Gomes de Araújo, João Marcos Teixeira Lacerda, Marcel da Câmara Ribeiro Dantas, Hélio Roberto Hékis, Ricardo Alexsandro de Medeiros Valentim

Resumo Uma identificação correta de transientes em sinais de ECG (Eletrocardiograma) pode auxiliar métodos de processamento de sinal de ECG, pois esse tipo de evento degrada o sinal e pode induzir a erros. Diante disso, o presente trabalho propõe uma arquitetura para a detecção desses fenômenos, seguindo a tendência atual da computação distribuída, na qual um sensor realiza a detecção dos transientes no momento da aquisição do sinal, e, em seguida, encaminha essa informação através de uma rede de comunicação de dados, desenvolvida especialmente para a automação hospitalar, até um dispositivo computacional que irá processar os dados ou então apresentá-los a um profissional capacitado para fazer a análise de forma manual. Para realizar a detecção de transientes, foi proposto um método matemático baseado na transformada Hilbert do sinal de ECG, aliado ao PM-AH (Protocolo Multiciclos para Automação Hospitalar), com adição de quadros neste, para que seja possível o envio da informação sobre a ocorrência de transientes junto aos dados do sinal de eletrocardiograma. Dentre os transientes possíveis, foi escolhido o ruído, por ser o fenômeno que mais interfere no processamento de sinais de ECG, onde testes foram realizados com a base de dados MIT-BIH Arrhythmia Database, enquanto uma análise matemática foi feita nos novos quadros do protocolo PM-AH, com o intuito de demonstrar a consistência do protocolo com esta adição.

Palavras-chave Transiente, ECG, Transformada Hilbert, PM-AH, Computação distribuída.

\section{An architecture for online transient detection in electrocardiogram signals on the MP-HA protocol}

\footnotetext{
Abstract A correct identification of transients in the ECG (electrocardiogram) can assist processing methods for ECG signals, since this type of event degrades the signal and can be misleading. Therefore, this paper proposes an architecture for detection of these phenomena, following the current trend of distributed computing, in which a sensor will detect transients at the time of signal acquisition, and then forward this information through a data communication network, designed specifically for hospital automation, to a computing device that will process the data or present it to a trained professional for manual analysis. To perform the detection of transients, a mathematical method based on the Hilbert transform of the ECG signal is proposed here, allied with the MP-HA (Multicycle Protocol for Hospital Automation), with the addition of frames, so that information on the occurrence of transients can be transmitted along with signal data of the electrocardiogram. Among the possible transients, noise was chosen because it is the phenomenon that interferes the most with the processing of ECG signals. Tests were performed using the MIT-BIH Arrhythmia Database, while a mathematical analysis was used in the new frames of the MP-HA protocol in order to demonstrate the consistency of the protocol with this addition.
}

Keywords Transients, ECG, Hilbert transform, MP-HA, Distributed computing. 


\section{Introdução}

Transiente é um termo utilizado em diversas áreas do conhecimento para designar um fenômeno de curta duração que interfere no funcionamento de um determinado sistema. Na cardiologia, esses fenômenos podem ser de origem morfológica (Biagini et al., 1981) ou ocorrer devido a eventos elétricos externos ao coração. Alguns tipos de ruídos/artefatos se encaixam na segunda categoria de transientes (Friesen et al., 1990) e diferentemente dos ruídos/artefatos de regime permanente, não podem ser filtrados analogicamente ou digitalmente, pois o espectro de frequência de alguns ruídos/artefatos sobrepõe-se com o espectro do próprio sinal de ECG (Sörnmo e Laguna, 2005), sendo necessário fazer uma filtragem adaptativa, ou utilizar detectores desenvolvidos especialmente para esse fim. A primeira etapa desse processo é a identificação de ruídos/artefatos transientes.

Os métodos de classificação de batimentos identificam transientes com base na duração do intervalo RR entre os batimentos cardíacos; Chazal et al., 2004), porém, essa abordagem baseada na morfologia do sinal não é capaz de detectar os transientes na forma de ruído/artefato, pois esses tipos de transientes não alteram a morfologia do sinal e sim a energia dele. Para esse fim, outra abordagem foi tomada no presente trabalho, a detecção de transientes com base na energia entre os batimentos cardíacos.

A tendência atual da computação é a distribuição do processamento entre as diversas partes do sistema de computação (Pedreiras et al., 2005). Isso decorre do desenvolvimento da eletrônica digital que possibilitou um aumento significativo na capacidade de processamento dos sensores, dessa forma, ganhando a denominação de smart sensors. Esses dois fatores tiveram um forte impacto no desenvolvimento da automação industrial nos anos recentes.

Os conceitos fundamentais da automação industrial (Nitzan e Rosen, 1976) são muitos mais abrangentes e não se limitam somente ao ambiente industrial. Tais conceitos podem ser aplicados na área médica, formando a automação hospitalar. Dentre os trabalhos publicados na área de automação hospitalar, destaca-se o protocolo de tempo-real multicast denominado de PM-AH (Protocolos Multiciclos para Automação Hospitalar) (Valentim et al., 2008) que será utilizado no presente trabalho.

O PM-AH é um protocolo de tempo-real que segmenta uma rede de dispositivos sobre o protocolo da camada de enlace IEEE 802.3 em grupos multicast IGMP (Internet Group Managment Protocol) Snooping, onde um leito hospitalar é associado a um grupo multicast, e um grupo multicast a vários dispositivos médicos, e todos esses grupos são gerenciados por um Provedor de Serviços (PS) através da estrutura mestre-escravo. A utilização de grupos multicast otimiza o desempenho da rede, pois evita que mensagens de um grupo interfiram em outro grupo. Cada grupo multicast divide a transmissão de mensagens em dois ciclos temporais, um ciclo síncrono, no qual os dispositivos podem enviar mensagens de sincronização e dados referentes aos sensores biomédicos através da estrutura de passagem de token e um ciclo assíncrono, de tal maneira que qualquer dispositivo pode enviar uma mensagem de controle ou alarmes.

As mensagens de dados, controle e alarmes são enviadas para o PS, elemento centralizador da rede. Além dessa função, o PS também verifica a conectividade dos sensores, notificando quando necessário, a ocorrência de falha de conectividade de algum sensor. A tolerância à falha também é abordada no protocolo, que prevê o uso de nós redundantes para lidar com esse aspecto. Uma visão geral do funcionamento do PM-AH pode ser visto na Figura 1.

Com relação à pilha de protocolos, o $\mathrm{PM}-\mathrm{AH}$ define as camadas de transporte, rede e enlace da pilha de protocolo TCP/IP. Para a camada de transporte, o PM-AH utiliza o UDP (User Datagram Protocol) que não emprega o conceito de confirmação do recebimento de dados e apresenta um desempenho melhor para transmissão de dados em tempo-real. Na camada de rede é utilizado o protocolo IP e o protocolo IGMP, já na camada de enlace, o protocolo IEEE 802.3, que oferece suporte ao IGMP snooping. A pilha de protocolos do PM-AH pode ser vista na Figura 2.

\section{Materiais e Métodos}

Pelo teorema fundamental da amostragem, a frequência de amostragem deve ser pelo menos duas vezes maior que a frequência fundamental do sinal. $\mathrm{O}$ sinal de ECG tem informações relevantes até $100 \mathrm{~Hz}$, o que faz com que uma frequência de $200 \mathrm{~Hz}$ seja a menor possível para aferir um sinal de ECG, de fato, muitos eletrocardiógrafos utilizam frequências maiores por diversos motivos como, por exemplo, utilização de filtros ou melhorar a resolução do sinal (os sinais de ECG utilizados nesse trabalho possuem uma frequência de amostragem de $360 \mathrm{~Hz}$ ). Esse valor é bastante baixo quando comparado à tecnologia atual de sistemas embarcados, o que faz com que o dispositivo de processamento passe muito tempo ocioso caso ele realize somente o envio de dados em uma rede de computadores. A presente arquitetura aproveita esse tempo ocioso para realizar um pré-processamento no sinal e fazer a detecção de transientes, para em seguida, enviar essa informação, através do protocolo PM-AH, para o PS, para este realizar o processamento do sinal. Alguns exemplos de processamentos que podem ser realizados são: 
- Identificar ruídos nos transientes e eliminar dados que possam interferir no processamento de métodos para identificação de patologias; e

- Identificar a ocorrência de batimentos de marca-passo.

O pré-processamento realizado se divide em três etapas:

- Extração de parâmetros;

- Avaliação da energia entre os parâmetros extraídos; e

- Formação de quadros do PM-AH.

A primeira etapa visa identificar um batimento cardíaco de forma única. Para isso é necessário escolher um parâmetro. Dentre os parâmetros, os pontos fiduciais são os melhores candidatos, pois são pontos relevantes usados para mapear um sinal de ECG. Dentre esses pontos, foi escolhida a onda $\mathrm{R}$, por ser o parâmetro mais largamente pesquisado por apresentar uma amplitude maior com relação aos demais. Para a extração das ondas R do sinal de ECG foi utilizado o trabalho de Hamilton e Tompinks (1986). A escolha desse trabalho dentre os demais se deu a:

- Alto desempenho: possui sensibilidade de 99,69\% e predição positiva de 99,77\%; e

- Baixo custo computacional: o algoritmo é implementado utilizando ferramentas de baixo custo computacional como derivada, raiz quadrada, somatório e mediana.

Uma visão geral da arquitetura pode ser visto na Figura 3.

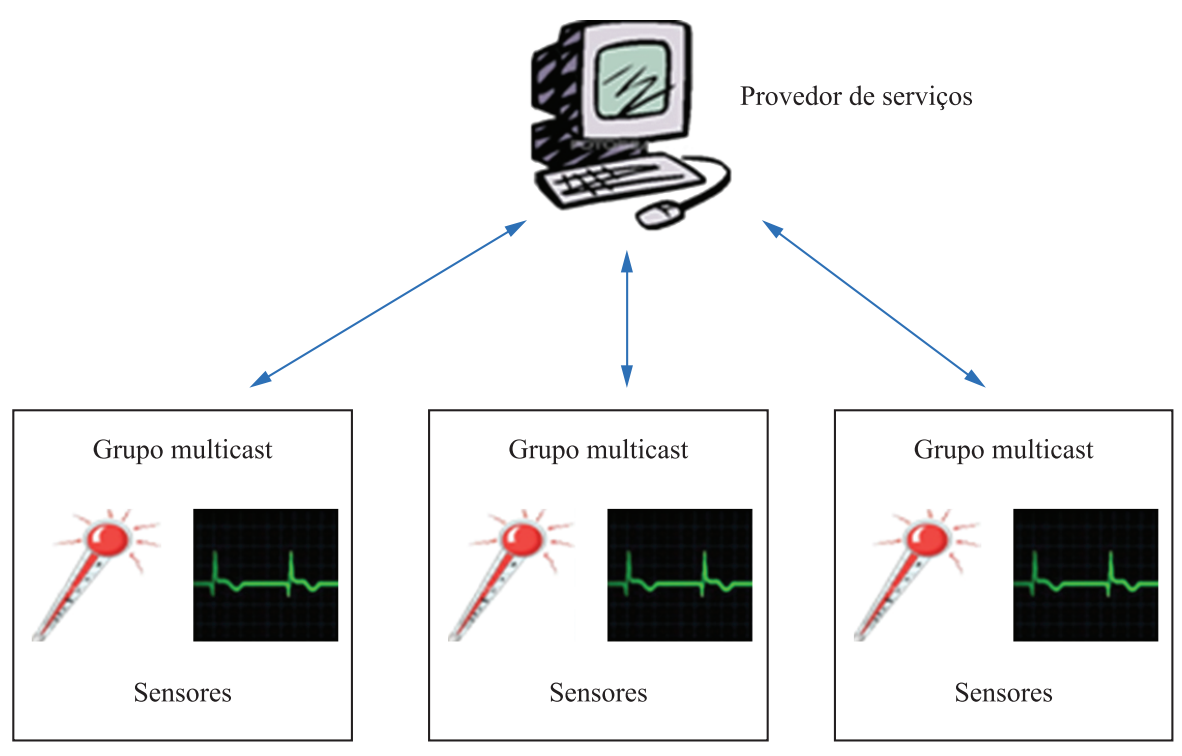

Figura 1. Visão geral do protocolo PM-AH. Figure 1. Overview of the MP-HA Protocol.

\section{Camada de aplicação}

\section{UDP}

IP multicasting

IEEE 802.3/IGMP snooping
PM-AH 


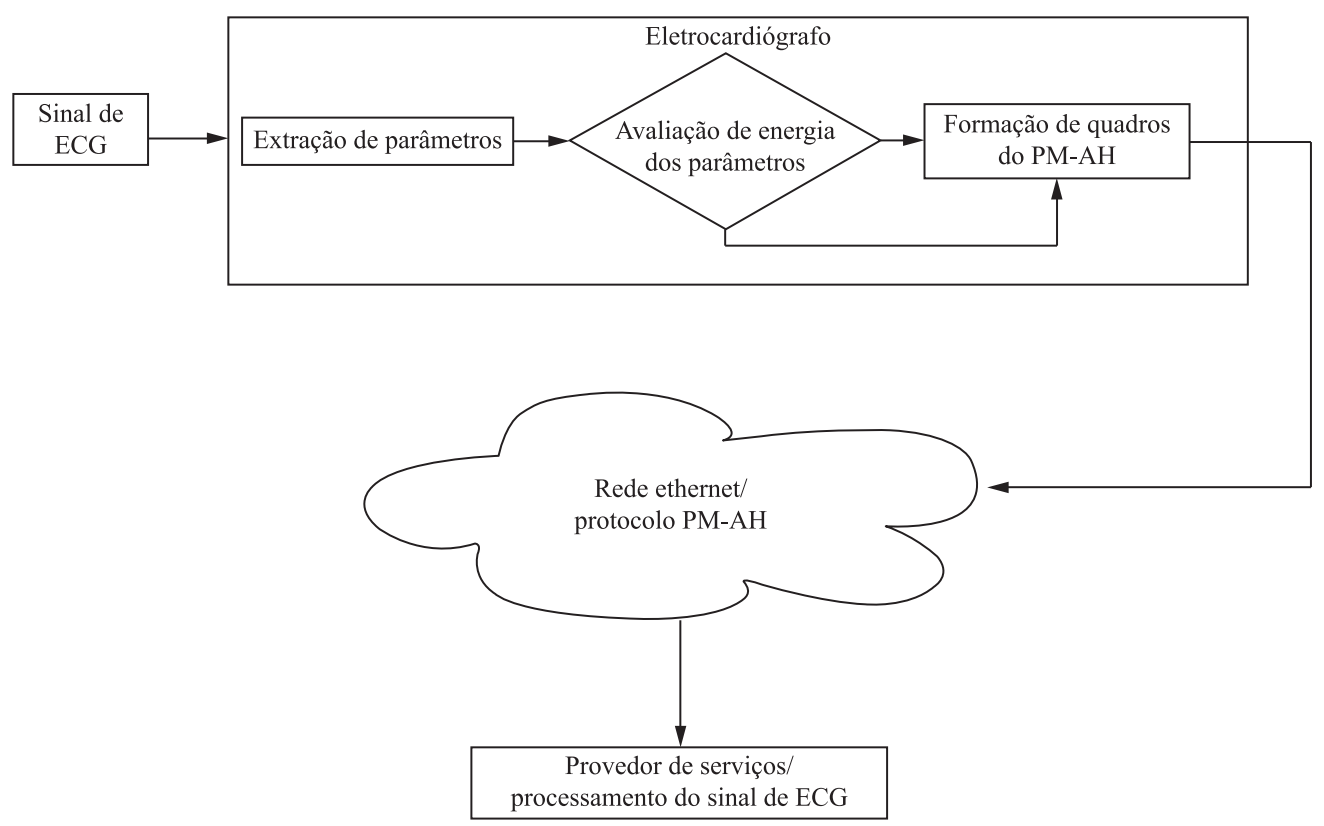

Figura 3. Visão geral da arquitetura.

Figure 3. Architecture Overview.

\section{Avaliação de Energia entre os Parâmetros Extraídos}

Uma vez que o parâmetro escolhido tenha sido extraído do sinal de ECG (i.e. onda R), a arquitetura propõe que seja aplicada a transformada Hilbert (Johansson, 1999) no sinal de ECG. A escolha dessa transformada foi feita devido à sua propriedade de ser uma função ímpar, ou seja, ela cruzará o eixo das abscissas (ou ela fará um cruzamento de zeros, ou uma inversão de sinal) toda vez que ocorrer um ponto de inflexão no sinal original. Semelhantemente, uma sucessão entre pontos de inflexão positivos e negativos será representada como um pico no conjugado da transformada Hilbert (Benitez et al., 2001). Assim, a energia do envelope do sinal sofrerá um aumento significativo devido a pequenas alterações no sinal. Essa propriedade é interessante para a deteç̧ão de transientes em sinais no tempo. Isso pode ser observado na Figura 4.

O próximo passo é o cálculo da energia entre as ondas-R do módulo do sinal analítico do sinal de ECG. O sinal analítico é dado pela Equação 1:

$g+(t)=g(t)+j \hat{g}(t)$

onde $\mathrm{g}(\mathrm{t})$ é o sinal no tempo e $\mathrm{g}(\mathrm{t})$ é a transformada Hilbert do sinal. A ideia principal por trás desse método é que quando ocorrer um ruído ou artefato, o módulo do sinal analítico será bastante modificado $\mathrm{e}$, consequentemente, sua energia será diferente do restante do sinal. Não é somente o ruído/artefato que é capaz de causar esse tipo de alteração no sinal, outros fenômenos podem fazê-lo, como por exemplo:

- Erro no algoritmo de detecção de ondas R;

- Distúrbios de condução causados por medicamentos; e

- Fenômenos não-estacionários:

- Histerese do coração (Clifford et al., 2006);

- Arritmias; e

- Respiração.

Por isso, a arquitetura propõe a detecção e não a identificação do ruído uma vez que uma alteração na energia entre as ondas-R não implica necessariamente em dizer que ocorreu um ruído/artefato.

Para obter melhores resultados foi feito um refinamento do sinal gerado pela energia entre as ondas-R do envelope do sinal de ECG (SEOR-ECG). Observações experimentais mostraram que a alteração na energia causada por ruídos é maior que os demais fenômenos, o que provoca uma inclinação acentuada no SEOR-ECG. Portanto, foi realizada a derivação do sinal.

A detecção de um transiente é realizada através de um limiar, caso um valor de energia supere esse limiar, ele é classificado como transiente. Depois de numerosos testes, esse limiar foi definido como sendo $10 \times$ o valor do maior intervalo entre os oitos primeiros intervalos. A Figura 5 mostra que a energia no intervalo RR do envelope do sinal de ECG é alterada de forma bastante significativa quando ocorre um transiente. 

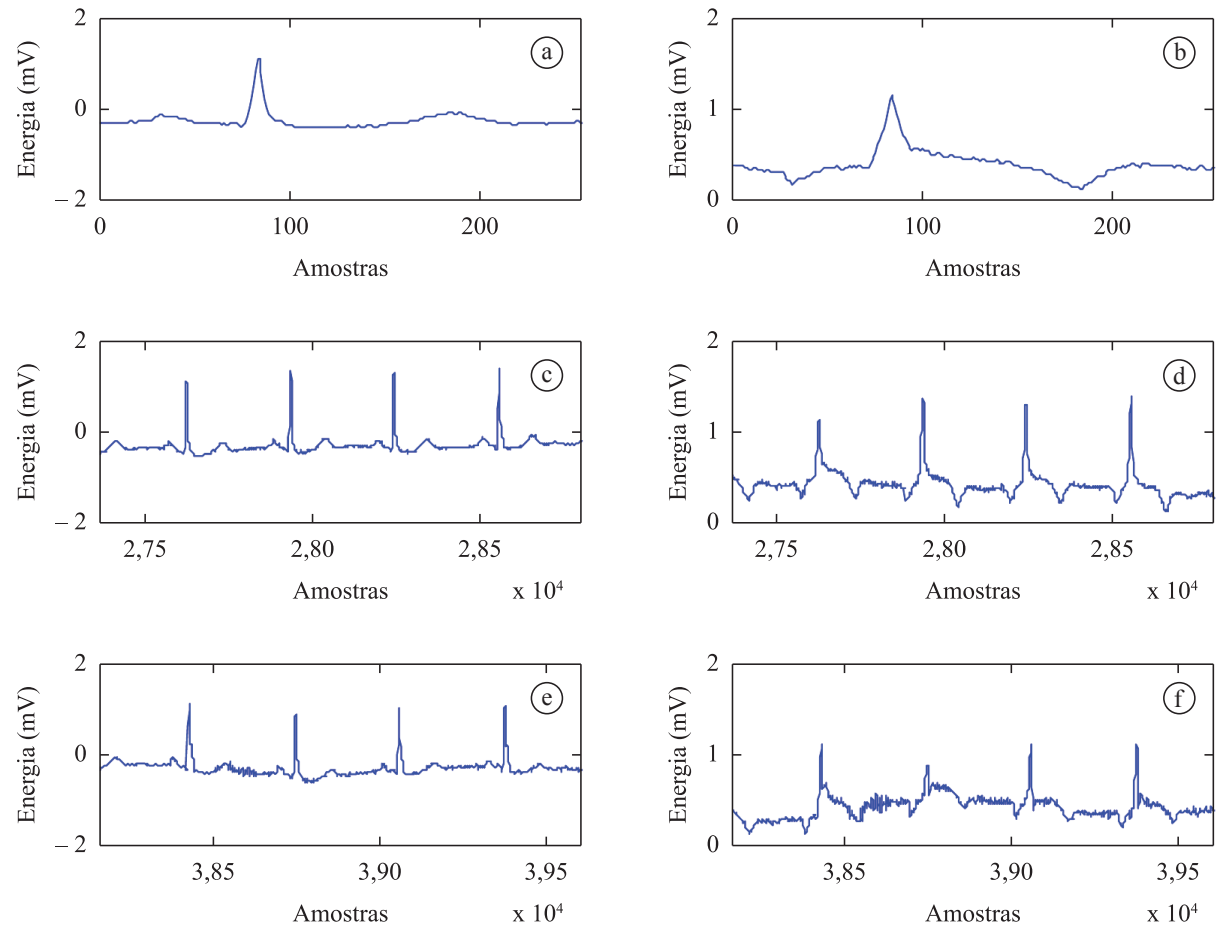

Figura 4. Viabilidade da Transformada Hilbert: a) um ciclo de sinal ECG; b) seu envelope equivalente; c) vários ciclos de sinal ECG sem ruído; d) seu envelope equivalente; e) vários ciclos de sinal ECG contaminados por ruído; f) seu envelope equivalente.

Figure 4. Viability of the Hilbert transform: a) an ECG cycle; b) the respective envelope; c) various ECG cycles without noise; d) the respective envelope. e) various ECG cycles with noise; f) the respective envelope.
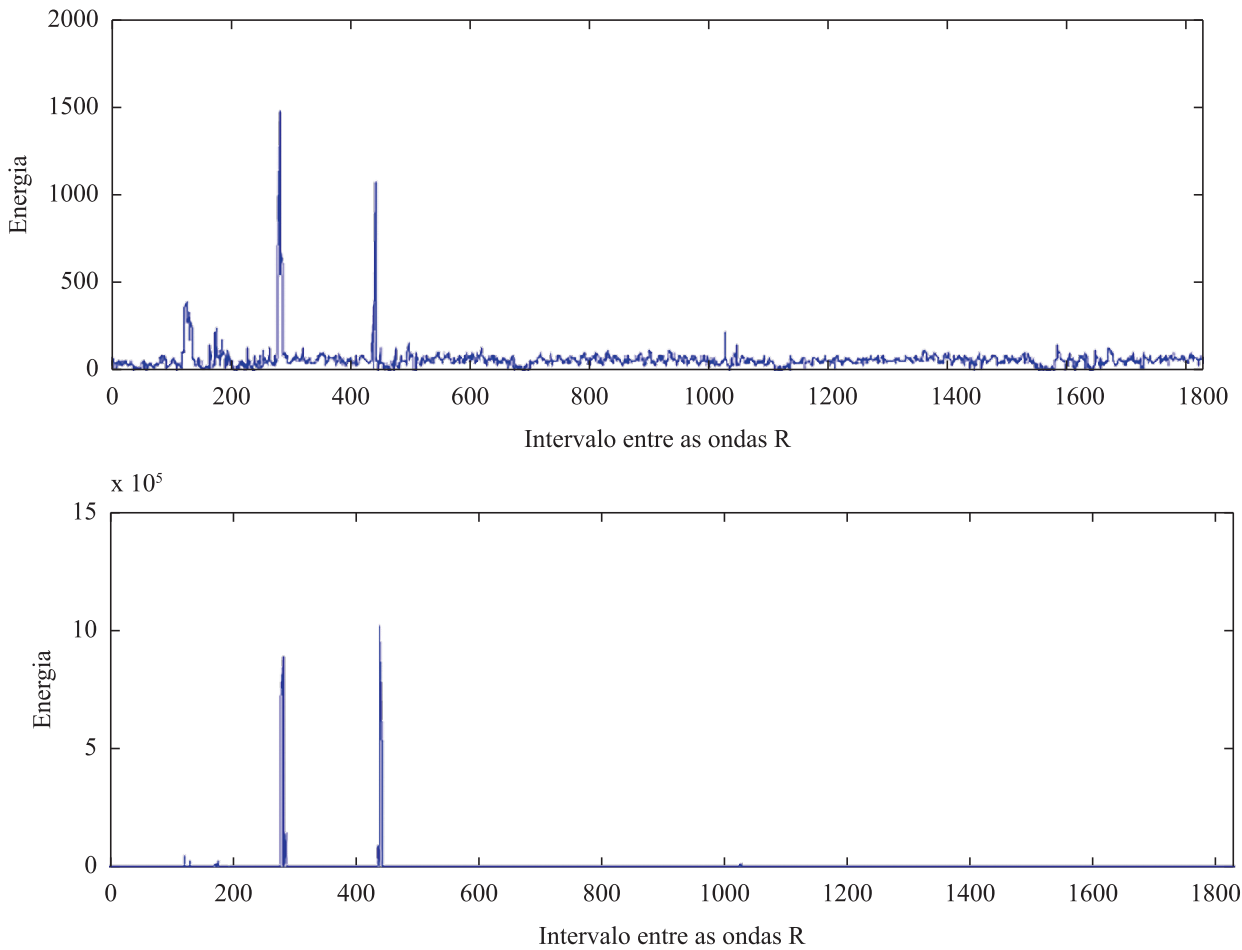

Figura 5. Valor da energia entre as ondas R (superior) e valor da energia derivado e elevado ao quadrado (inferior).

Figure 5. Value of the energy between $R$ waves and value of the energy derivative and squared. 


\section{Quadros do Protocolo PM-AH para Indicar a Ocorrência de Transientes}

Quando a energia de um intervalo do SEOR-ECG ultrapassa o limiar, o PS do protocolo PM-AH precisa ser notificado desse evento, para isso, a arquitetura propõe a criação de novos quadros, o BEAT_MESSAGE e o BEAT_CONFIRM_MESSAGE. Esses novos quadros são enviados no ciclo assíncrono do protocolo para que a transmissão dos dados de ECG não seja afetada e um não-determinismo seja criado na rede.

A mensagem BEAT_MESSAGE é enviada quando o limiar é ultrapassado e tem o formato mostrado na Figura 6.

A mensagem BEAT_CONFIRM_MESSAGE é enviada pelo PS para indicar o recebimento da mensagem BEAT_MESSAGE que pode se perder na rede, sendo necessário prover um mecanismo de confirmação. A mensagem BEAT_CONFIRM_MESSAGE é utilizada para essa finalidade e tem o formato mostrado na Figura 7.

O mecanismo de confirmação proposto pela arquitetura afirma que quando uma mensagem BEAT_MESSAGE é enviada em um ciclo assíncrono, uma mensagem de confirmação deve ser enviada no ciclo assíncrono seguinte. Caso isso ocorra, a mensagem foi entregue corretamente. Caso isso não ocorra, uma nova mensagem BEAT_MESSAGE deve ser enviada no ciclo assíncrono seguinte com o campo "atraso" acrescido de 2 (relativo ao ciclo assíncrono da confirmação não recebida e o ciclo atual). O funcionamento pode ser visualizado na Figura 8.

8

40

56

64

\begin{tabular}{|l|l|l|l|}
\hline Tipo & Endereço & Porta & Atraso \\
\hline
\end{tabular}

\begin{tabular}{|c|c|c|}
\hline Campo & Tamanho & Descrição \\
\hline Tipo & 8 bits & $\begin{array}{c}\text { Tipo de mensagem que } \\
\text { está sendo enviada. }\end{array}$ \\
\hline Endereço & 32 bits & $\begin{array}{c}\text { Endereço multicast do } \\
\text { grupo que vai receber a } \\
\text { confirmação. }\end{array}$ \\
\hline Porta & 16 bits & $\begin{array}{c}\text { Campo que identifica o } \\
\text { dispositivo que está } \\
\text { aguardando a resposta. }\end{array}$ \\
\hline Atraso & 8 bits & $\begin{array}{c}\text { Indica a quantidade de } \\
\text { ciclos desde que ocorreu o } \\
\text { último batimento. }\end{array}$ \\
\hline
\end{tabular}

Figura 6. Quadro BEAT_MESSAGE. Formato e descrição dos campos.

Figure 6. BEAT_MESSAGE Frame. Format and description of the fields.

\begin{tabular}{|c|c|c|}
\hline \multicolumn{2}{c|}{8} & 56 \\
\hline Tipo & Endereço & Porta \\
\hline Campo & Tamanho & Descrição \\
\hline Tipo & 8 bits & $\begin{array}{c}\text { Campo destinado a } \\
\text { armazenar o tipo de } \\
\text { mensagem que está } \\
\text { sendo enviado }\end{array}$ \\
\hline Endereço & 32 bits & $\begin{array}{c}\text { Endereço multicast do } \\
\text { grupo que vai receber a } \\
\text { confirmação }\end{array}$ \\
\hline Porta & 16 bits & $\begin{array}{c}\text { Campo que identifica o } \\
\text { dispositivo que está } \\
\text { aguardando a resposta }\end{array}$ \\
\hline
\end{tabular}

Figura 7. Quadro BEAT_CONFIRM_MESSAGE. Formato e descrição dos campos.

Figure 7. BEAT_CONFIRM_MESSAGE Frame. Format and description of the fields. 


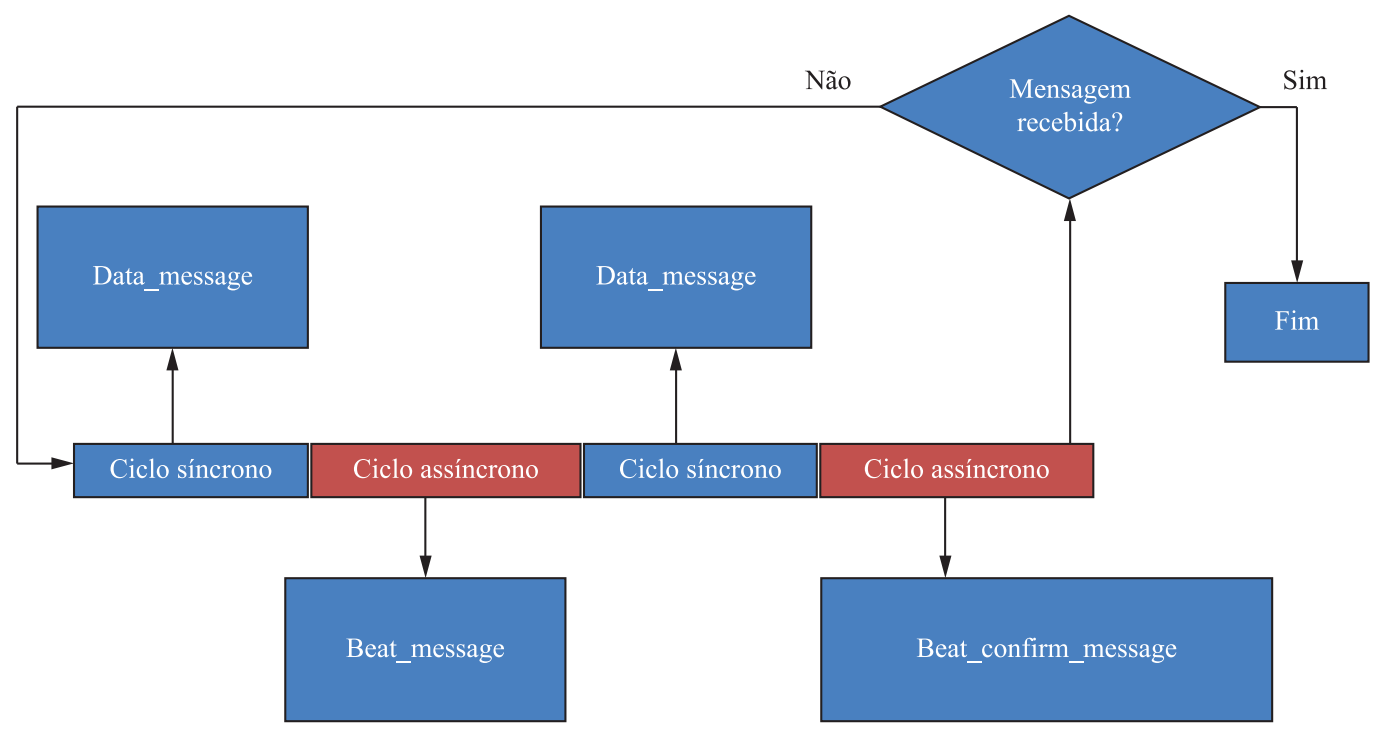

Figura 8. Funcionamento dos novos quadros.

Figure 8. Operation of the new frames.

O campo "atraso" indica a localização do transiente, uma vez que todo ciclo síncrono é intercalado por um ciclo assíncrono, sendo então possível fazer uma busca retrógrada e determinar o momento da ocorrência do transiente. Esse campo deve levar em consideração o atraso provocado pela janela móvel do algoritmo de extração de parâmetros e atrasos computacionais.

\section{Resultados}

Testes foram realizados com ECG de pacientes do MIT/BIH Arrythmia Database, uma das bases de dados mais utilizada na pesquisa de processamento de sinais de ECG (Massachusetts..., 1989; Moody e Mark, 2001). Foram selecionados os ECG que apresentam ruídos e artefatos no sinal totalizando 35 eventos, dos quais 32 foram detectados, ou seja, $91,42 \%$ dos ruídos foram detectados e os transientes identificados pelo método correspondem a 6,24\% do sinal. Diminuindo drasticamente a quantidade de sinal que seria necessário processar por um método de identificação de ruídos. Os dados encontram-se na Tabela 1.

\section{Discussão}

Uma questão em aberto na arquitetura proposta neste trabalho é a determinação do limiar. Nos testes, foi utilizado um valor experimental cujo os resultados obtidos foram considerados satisfatório, o que prova a viabilidade da utilização da energia da transformada Hilbert no intervalo R-R para a detecção de transientes, porém, não existe uma garantia de que esse seja o valor ótimo. Isso é um tema para trabalhos futuros.
Outro aspecto a ser discutido é o impacto dos novos quadros no protocolo PM-AH., pois quaisquer alterações no protocolo devem preservar as suas características de tempo-real. Ambos os quadros utilizam a janela assíncrona do protocolo que, por padrão, é igual à janela síncrona. A menor janela síncrona possível ocorre quando somente um dispositivo esteja transmitindo. Como, por padrão, a janela síncrona tem a mesma duração da janela assíncrona, a menor janela assíncrona possível tem o tamanho de uma mensagem de dados ( 88 bits).

Isso pode ser inferido da seguinte fórmula (Tanenbaum, 2003):

$$
T_{j s}=\sum_{m=1}^{q}\left(A P_{m}+A T_{m}\right), A P=(d / v p m f), A T=L / R
$$

onde:

- $\mathrm{T}_{\mathrm{js}}=$ tempo de uma janela síncrona:

- $\mathrm{q}=$ quantidade de nós inscritos no grupo (considerado o nó mestre); e

- $\mathrm{m}=$ mensagem enviada.

- $\mathrm{AP}=$ atraso de propagação do sinal no meio físico:

- d = distância do enlace; e

- $\operatorname{vpmf}=$ velocidade de propagação no meio físico.

- $\mathrm{AT}=$ atraso da transmissão:

- $\mathrm{L}=$ comprimento da mensagem (em bits); e

- $\mathrm{R}$ = banda do enlace (taxa de transmissão em bits/s). 
Tabela 1. Desempenho da Transformada Hilbert na detecção de ruídos.

Table 1. Performance of the Hilbert transform to detect noise.

\begin{tabular}{ccccc}
\hline Pacientes & $\begin{array}{c}\text { Quantidade de } \\
\text { ondas-R }\end{array}$ & $\begin{array}{c}\text { Transientes } \\
\text { identificados }\end{array}$ & $\begin{array}{c}\text { Quantidade de } \\
\text { ruídos }\end{array}$ & $\begin{array}{c}\text { Quantidade de } \\
\text { ruídos identificados }\end{array}$ \\
\hline 101 & 1859 & 34 & 2 & 2 \\
104 & 2224 & 42 & 2 & 2 \\
105 & 2564 & 369 & 6 & 6 \\
107 & 2131 & 60 & 1 & 0 \\
109 & 2526 & 12 & 2 & 4 \\
111 & 2120 & 357 & 4 & 1 \\
119 & 1985 & 15 & 2 & 2 \\
200 & 2595 & 41 & 2 & 2 \\
203 & 2976 & 175 & 2 & 2 \\
209 & 2999 & 180 & 2 & 2 \\
210 & 2645 & 39 & 2 & 1 \\
212 & 2746 & 76 & 2 & 0 \\
217 & 2202 & 141 & 1 & 1 \\
221 & 2422 & 14 & 1 & 2 \\
222 & 2492 & 118 & 1 & 1 \\
234 & 2048 & 678 & 2 & \\
\hline
\end{tabular}

Pacientes: Número do paciente no MIT/BIH Arrythmia Database. Quantidade de ondas-R: ondas que foram detectadas pelo método de Hamilton-Tompkins. Transientes identificados: batimentos que foram identificados como transientes pelo método. Quantidade de ruídos: quantidade de ruídos que foram identificados pelo MIT/BIH Arrythmia Database. Quantidade de ruídos identificados: ruídos que foram identificados corretamente pelo método.

O único fator a ser analisado é o tamanho das mensagens, porque os demais parâmetros permanecem os mesmos para ambas as janelas. Uma mensagem BEAT_MESSAGE e uma BEAT_CONFIRM_MESSAGE têm respectivamente 64 bits e 56 bits, portanto, são menores que uma mensagem de dados. Aspecto esse que garante que não haverá interferência no comportamento do protocolo, visto que o tamanho da mensagem tem relação direta como o tempo de propagação do sinal no meio físico, ou seja, na latência.

Um aspecto importante a ser destacado referente ao objeto de pesquisa desse artigo, foi o estudo sistematizado em busca de métodos que tivessem correlação com trabalho desenvolvido, ou seja, que apresentassem mecanismos de identificação de transientes em sinais de ECG. Diante de tais estudos, observou-se que há originalidade na temática abordada neste artigo, pois não foram encontrados na literatura estudos cujo objetivo era a detecção de transientes em sinais de eletrocardiograma. Neste ponto especificamente, o artigo apresente uma contribuição importante, por desenvolver uma metodologia original baseada na Transformada Hilbert para compor uma arquitetura de comunicação em tempo real para detecção online de transientes em sinais de eletrocardiograma. Deste modo, apontando um novo campo que poderá ser ainda mais explorado quanto ao processamento de sinais de eletrocardiograma, por exemplo, desenvolvendo novas pesquisas que possibilitem realizar estudos comparativos para identificar métodos mais eficientes para detecção de transientes.

\section{Conclusão}

A ocorrência de transientes, especialmente os que se apresentam sob a forma de ruído/artefato, interfere nos métodos matemáticos para processamento de sinal de ECG. Devido ao fato de não ser um evento previsível, é necessário fazer uma filtragem adaptativa de transiente para evitar a eliminação de informação útil do sinal, sendo a detecção da ocorrência desse evento, o primeiro passo para a sua eliminação.

Com base nos estudos desenvolvido e nos resultados alcançados, verificou-se que a arquitetura desenvolvida pode se constituir como uma ferramenta bastante atraente no pré-processamento de eliminação de transientes em sinais de ECG, uma vez que utiliza métodos matemáticas de baixo custo computacional, tanto na extração de parâmetros como na detecção de transientes, e cria novos quadros para o protocolo $\mathrm{PM}-\mathrm{AH}$ de forma que sua característica de tempo-real permanece inalterada. Essas duas características associadas fazem da arquitetura abordada nesse artigo uma ferramenta computacional simples e eficiente no pré-processamento de sinal de ECG. 


\section{Agradecimentos}

Ao CNPq pelo financiamento da pesquisa e ao Laboratório de Inovação Tecnológica em Saúde (LAIS) do Hospital Universitário Onofre Lopes (HUOL) da Universidade Federal do Rio Grande do Norte (UFRN) por ter concedido a infraestrutura necessária a pesquisa.

\section{Referências}

Biagini A, Mazzei MG, Carpeggiani C, Testa R, Antonelli R, Michelassia C, L'Abbate A., Maseri A. Vasospastic ischemic mechanism of frequent asymptomatic transient ST-T changes during continuous electrocardiografic monitoring in selected unstable angina patients. American Heart Jornal. 1981; 103(1):13-20. http://dx.doi. org/10.1016/0002-8703(82)90523-3

Chazal P, O’Dwyer M, Reilly RB. Automatic classification of heatbeats using ECG morphology and heartbeat interval features. IEEE Transactions on Biomedical Engineering. 2004; 51(7):1196-206. PMid:15248536. http://dx.doi.org/10.1109/TBME.2004.827359

Clifford GD, Azuaje F, McSharry PF. Advanced methods and tools for ECG data analysis. Cambridge; 2006.

Friesen GM, Jannett TC, Jadallah MA, Yattes SL, Quint SR, Nagle HT. A comparison of the noise sensitivity of nine QRS detection algorithms. IEEE Transactions on Biomedical Engineering. 1990; 37(1):85-97. http://dx.doi. org/10.1109/10.43620

Hamilton PS, Tompkins WJ. Quantitative investigation of QRS detection rules using the MIT/BIH Arrhythmia Database. IEEE Transactions on Biomedical Engineering. 1986; 33(12):115765. http://dx.doi.org/10.1109/TBME.1986.325695
Johansson M. The Hilbert Transform [thesis]. Växjö: Växjö University; 1999.

Benitez D, Gaydecki PA, Zaidi A, Fitzpatrick AP. The use of the Hilbert Transform in ECG signal analysis. Computers in Biology and Medicine. 2001; 31(5):399-406. http://dx.doi. org/10.1016/S0010-4825(01)00009-9

Massachusetts Institute of Technology - MIT. MIT-BIH Arrhythmia Database. 1989. [cited 2011 May 10]. Available from: http://www.physionet.org/physiobank/database/html/ mitdbdir/mitdbdir.htm.

Moody GB, Mark RG. The impact of the MIT Arrhythmia Database. IEEE Engineering in Medicine and Biology. 2001; 20(3):45-50. http://dx.doi. org/10.1109/51.932724

Nitzan D, Rosen CA. A programable industrial automation. Transactions on Computers. 1976; 25(12):1259-70. http:// dx.doi.org/10.1109/TC.1976.1674593

Pedreiras P, Almeida L, Gal P, Giorgio B. FFT-Ethernet: A flexible real-time communication protocol that supports dynamic QoS management on Ethernet-based systems. IEEE Transactions on Industrial Informatics. 2005; 1(3):162-72. http://dx.doi.org/10.1109/TII.2005.852068

Sörnmo L, Laguna P. Bioelectrical signal processing in cardiac and Neurological applications. London: Elsevier; 2005.

Tanenbaum AS. Redes de computadores. 4. ed. Prentice-Hall; 2003.

Valentim RAM, Morais AHF, Brandão GB, Guerreiro AMG, Xavier MA, Araújo Junior HB, Bezerra HU, Florentino GH, Paz de Araújo CA. MP-AH: Multicycles protocol for hospital automation over multicast addressing. In: INDIN 2008: Proceedings of the 6th IEEE International Conference on Industrial Informatics; 2008 July 13-16; Daejeon. Daejeon; 2008. p. 979-84. http://dx.doi.org/10.1109/ INDIN.2008.4618244

\footnotetext{
Autores

Diego Rodrigues de Carvalho*, Bruno Gomes de Araújo, João Marcos Teixeira Lacerda, Marcel da Câmara Ribeiro Dantas, Hélio Roberto Hékis, Ricardo Alexsandro de Medeiros Valentim Laboratório de Inovação Tecnológica em Saúde, Programa de Pós-graduação em Engenharia Elétrica e Computação - PPGEEC, Hospital Universitário Onofre Lopes - HUOL, Universidade Federal do Rio Grande do Norte - UFRN,

Av. Nilo Peçanha, 620, Petrópolis, CEP 59012-300, Natal, RN, Brasil.
} 